\title{
Biological proteins knock nanoparticles off target
}

Achieving optimal accumulation of nanoparticle-based drugs at the desired target is problematic, even though targeting strategies using antibodies or specific ligands are available.

A recent paper in Nature Nanotechnology offers a possible explanation as to why accumulation may be low. Dawson and colleagues showed that the proteins present in biological serum can shield targeted nanoparticles from their intended target.

To investigate the fate of targeted nanoparticles, the authors used fluorescent silica nanoparticles coated with polyethylene glycol polymer chains, which were used to attach the potential targeting protein transferrin. Because transferrin receptors are overexpressed on cancer cells as a result of iron metabolism, this approach has been commonly used in the development of targeted anticancer nanoparticles.

Initial experiments carried out in buffer solutions or serum-free medium established that these nanoparticles bound to and were internalized by the transferrin receptor on lung epithelial cells. However, studies using cells in which expression of the transerrin receptor had been silenced showed that some cellular uptake (around 30\%) was not dependent on the transferrin receptor, suggesting that targeted uptake rather than total uptake levels - is important in determining the specificity of nanoparticle-cell interactions.

To see whether targeting was preserved in more physiological conditions, the authors next

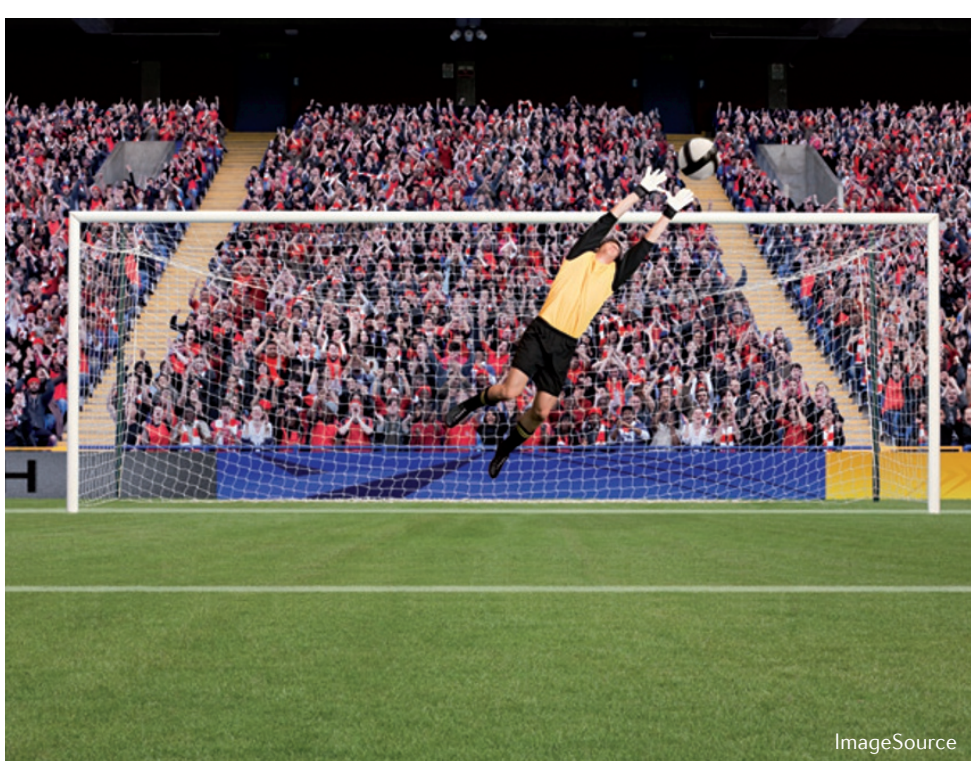

performed studies in solutions that contained bovine or human serum. They found that increasing the serum concentration $(0 \%, 10 \%$ or $55 \%$ ) reduced the interaction between the nanoparticles and the transferrin receptor. In addition, the proportion of transferrin receptor-mediated cellular uptake decreased - dropping to negligible levels - suggesting that the nanoparticles had completely lost their specificity.

Next, the authors investigated whether the binding of serum proteins to the nanoparticle (forming what is known as a protein corona around the nanoparticle) mediated the loss of specificity.

Indeed, protein binding that led to loss of target specificity was observed, even though the authors tried to reduce non-specific binding by attaching secondary polyethylene glycol to the nanoparticle surface or to transferrin.

So this study shows that serum caused targeted nanoparticles to lose specificity as a result of the formation of a protein corona around the nanoparticles. Although additional factors - such as the route of administration, tissue accumulation and cellular trafficking - probably contribute to the low targeted uptake, a step forward in the development of targeted nanoparticles would be to conduct in vitro studies in biologically relevant solutions to establish that their targeting properties are observed.

Charlotte Harrison

ORIGINAL RESEARCH PAPER Salvati, A. et al. Transferrin-functionalized nanoparticles lose their targeting capabilities when a biomolecule corona adsorbs on the surface. Nature Nanotech. 8 , 137-143 (2013) 